San Jose State University

SJSU ScholarWorks

Faculty Publications, Chemistry

Chemistry

$1-1-2002$

\title{
Temperature and Pressure Dependence of Excitation Spectra as a Probe of the Solution Structure and Equilibrium Thermodynamics of a Eu(III) Complex Containing a Modified Dota Ligand
}

Gilles Muller

San Jose State University, gilles.muller@sjsu.edu

S. D. Kean

D. Parker

J. P. Riehl

Follow this and additional works at: https://scholarworks.sjsu.edu/chem_pub

Part of the Chemistry Commons

\section{Recommended Citation}

Gilles Muller, S. D. Kean, D. Parker, and J. P. Riehl. "Temperature and Pressure Dependence of Excitation Spectra as a Probe of the Solution Structure and Equilibrium Thermodynamics of a Eu(III) Complex Containing a Modified Dota Ligand" Journal of Physical Chemistry (2002): 12349-12355. https://doi.org/ 10.1021/jp021747+

This Article is brought to you for free and open access by the Chemistry at SJSU ScholarWorks. It has been accepted for inclusion in Faculty Publications, Chemistry by an authorized administrator of SJSU ScholarWorks. For more information, please contact scholarworks@sjsu.edu. 


\title{
Temperature and Pressure Dependence of Excitation Spectra as a Probe of the Solution Structure and Equilibrium Thermodynamics of a Eu(III) Complex Containing a Modified dota Ligand
}

\author{
Gilles Muller, ${ }^{\dagger}$ Susanna D. Kean, ${ }^{\ddagger}$ David Parker, ${ }^{\ddagger}$ and James P. Riehl ${ }^{*} \dagger$ \\ Department of Chemistry, University of Minnesota Duluth, Duluth, Minnesota 55812 and \\ Department of Chemistry, University of Durham, South Road, Durham, U.K.
}

Received: July 29, 2002; In Final Form: October 16, 2002

\begin{abstract}
The temperature and pressure dependence of the ${ }^{7} \mathrm{~F}_{0} \rightarrow{ }^{5} \mathrm{D}_{0}$ and ${ }^{7} \mathrm{~F}_{1} \rightarrow{ }^{5} \mathrm{D}_{0}$ excitation spectra for aqueous solutions of Eu(III) with racemic-tetra( $\alpha$-carboxyethyl)DOTA have been measured. Individual excitation and emission peaks have been assigned to square antiprism (SAP) and twisted square antiprism (TSAP) isomers of the complex, and the variation of relative peak areas as a function of temperature and pressure have been used to estimate the enthalpy and volume change associated with the isomer equilibrium. The effects of temperature on the initial state populations have been treated explicitly, although for the data presented, these effects are within the uncertainty of the measurement. The results show that the SAP isomer of Eu(ceDOTA) is the more compact, more ordered, and lower enthalpy structure, consistent with previous NMR experiments on the differences in the observed rate of water exchange for the isomeric species.
\end{abstract}

\section{Introduction}

Interest in the solution structure, solution dynamics, and excited-state energetics of aqueous lanthanide complexes is increasing due to the development of important lanthanide-based spin and luminescence probes of biological systems. ${ }^{1-3}$ Much of this recent work has been concerned with lanthanide(III) complexes with macrocyclic ligands, because these types of systems often possess large stability constants while allowing one some control of subtle, but important structural details. For example, Gd(III)-based MRI contrast agents require effective complexation of the metal ion, yet must allow solvent water molecules to penetrate the first or second coordination sphere..$^{3,4}$ The importance of structural isomerism on the rate of water exchange in macrocyclic complexes with potential as MRI contrast agents has also been studied recently. ${ }^{5-7}$ It has been shown, for example, that the rate of water exchange for the major solution isomer (square antiprism $=\mathrm{SAP}$ ) of lanthanide(III) complexes with modified dota ligands is much slower than that measured for the minor twisted square antiprism isomer (TSAP). ${ }^{7}$ A direct correlation between the proportion of TSAP isomer and the water exchange rate has also been observed. ${ }^{7}$

Of special interest to us is the explicit consideration of chirality in the design of lanthanide-based biological probes. Chiral lanthanide complexes have also been the topic of a recent review. ${ }^{8}$ Not only does the use of chiral lanthanide complexes offer the possibility of increased selectivity toward chiral biological systems, but also it allows for the exploitation of chiroptical spectroscopic measurements such as circular dichroism (CD) and circularly polarized luminescence (CPL) as

* Author to whom correspondence should be addressed.

$\dagger$ University of Minnesota Duluth.

$\doteqdot$ University of Durham. specific probes of chiral structure and structural change. CPL has been a particularly useful technique for the study of the structure and dynamics of complexes of $\mathrm{Eu}(\mathrm{III})$ and $\mathrm{Tb}(\mathrm{III})$, because many complexes of these ions are luminescent, and selected intraconfigurational $\mathrm{f} \leftrightarrow \mathrm{f}$ electronic transitions, that obey magnetic dipole selection rules, often show large net circular polarization. ${ }^{9}$

The work described here is concerned with the solution equilibrium between solution isomers of the complex formed between Eu(III) and RRRR/SSSS-tetra( $\alpha$-carboxyethyl)DOTA.

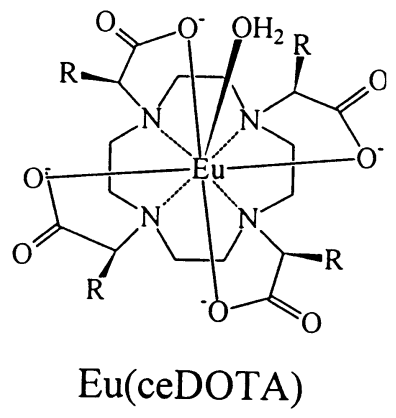

A water of hydration at the "cap" position is also illustrated in this drawing. The structure, NMR, and optical spectroscopy of this particular complex and related isomers have been studied previously. ${ }^{7}$ In particular, it has been shown that when the pendant arms attached to the ring nitrogens are not $\alpha$-substituted, arm rotation can lead to interconversion of TSAP and SAP isomers as well as a change in absolute configuration $(\Delta \leftrightarrow$ $\Lambda){ }^{6}$ However, for the complex studied here, and for other structurally related complexes, the only process that effectively interconverts these two isomers at ambient temperatures is 
cyclen ring inversion. This process is illustrated schematically below.

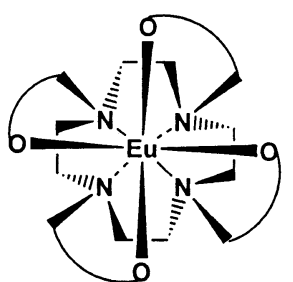

SAP
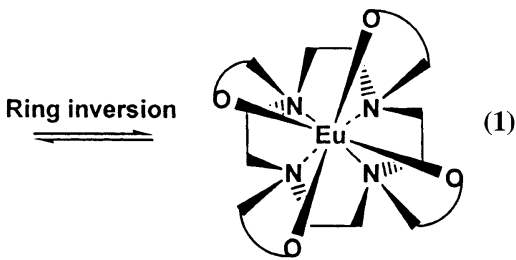

TSAP
In the work presented here, we present a number of wavelength selective excitation and emission measurements which are analyzed in terms of the structure and thermodynamics of the two isomers. Temperature and pressure dependence of the excitation spectra are presented and described, and comparison of the results reported here with previous work is given.

\section{Experimental Section}

$\mathrm{Eu}(\mathrm{III})$ complexes with 1,4,7,10-tetrakis( $\alpha$-carboxyethyl)1,4,7,10-tetraazacyclododecane [Eu(ceDOTA)] were synthesized as previously described. ${ }^{7}$ Substitution of the pendant arms of the dota ligand yields four chiral centers. The results presented here are for the complexes in which the chirality of all four chiral centers are the same (RRRR) or (SSSS). A $0.01 \mathrm{M}$ solution of [Eu(ceDOTA)] was prepared by dissolving weighed amounts of the solid compound in deuterated water, and the $\mathrm{pD}$ was adjusted to approximately 6 through the addition of $\mathrm{NaOH} / \mathrm{D}_{2} \mathrm{O}$. All measurements were performed in quartz fluorescence cuvettes with a path length of $0.4 \mathrm{~cm}$.

Excitation and emission measurements for the $\mathrm{Eu}(\mathrm{III}) \mathrm{com}$ plex were accomplished by using a Coherent-599 tunable dye laser $(0.03 \mathrm{~nm}$ resolution) with a Coherent Innova-70 argon ion laser as a pump source. The laser dye used in all measurements was rhodamine $6 \mathrm{G}$ dissolved in ethylene glycol. Calibration of the emission monochromator (and subsequently the dye laser wavelength) was accomplished by passing scattered light from a low-power $\mathrm{He}-\mathrm{Ne}$ laser through the detection system. The error in the dye-laser wavelength is assumed to equal the resolution of the emission monochromator $(0.1 \mathrm{~nm})$. The optical detection system consisted of a focusing lens, long pass filter, and $0.22 \mathrm{~m}$ monochromator. The emitted light was detected by a cooled EMI-9558B photomultiplier tube operating in photoncounting mode. Verification that the solution prepared from this ligand was indeed a racemic mixture was made by measuring the circularly polarized luminescence following circularly polarized excitation using an instrument described previously. ${ }^{10}$ Variation of the excitation polarization from right to left circular polarization produced an oppositely signed CPL result of equal magnitude as expected for a racemic mixture. For temperaturedependent experiments, the temperature of the sample was controlled by placing a $1.0 \mathrm{~cm}$ cuvette inside an anodized aluminum cell jacket which was connected to a NESLAB RTE101 circulating temperature bath, and monitored by placing the temperature probe (Fisher calibrated to $0.1{ }^{\circ} \mathrm{C}$ ) in the sample compartment before and after each measurement. The circulation fluid was water. The connections from the circulator to the aluminum cuvette jacket were insulated to help maintain consistent temperatures. Measurements of excitation spectra at greater than atmospheric pressure were accomplished in a custom-made stainless NOVA-SWISS high-pressure liquid cell with three sapphire windows. The sample capsule consisted of a modified $10 \mathrm{~mm}$ cylindrical glass tube fitted with an aluminum

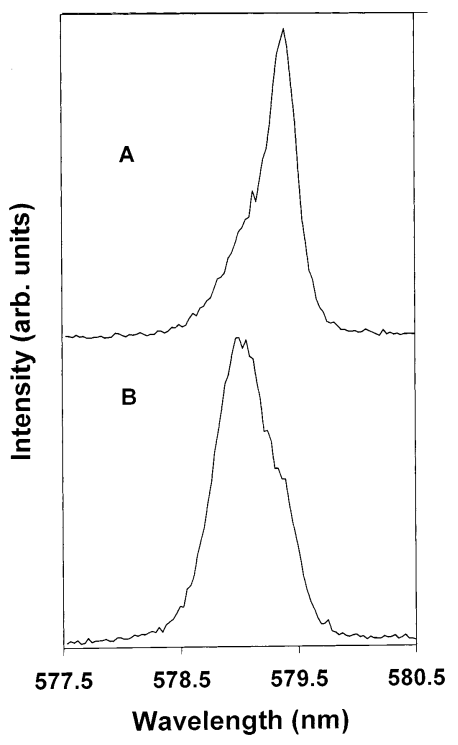

Figure 1. ${ }^{7} \mathrm{~F}_{0} \rightarrow{ }^{5} \mathrm{D}_{0}$ excitation spectra for a $0.01 \mathrm{M}$ aqueous solution of $\mathrm{Eu}(\mathrm{ceDOTA})$. The luminescence was monitored at $589.5 \mathrm{~nm}$ in (A) and $587 \mathrm{~nm}$ in (B).

piston with two O-rings. The solution sample is placed into the capsule, which is then placed inside the high-pressure cell block already filled with water, which acts as the hydraulic fluid. The high-pressure cell is then pressurized to a fixed static pressure and monitored using a Enerpac hydraulic hand pump and gauge. Before and after each excitation scan, the laser intensity was recorded in order to correct for any incident intensity fluctuations.

\section{Results}

As indicated above, an aqueous solution of Eu(ceDOTA) has been shown by NMR techniques to contain SAP and TSAP structures in approximately a 1:4 ratio. ${ }^{7}$ Evidence for the presence of two species in solution may also be obtained from high-resolution luminescence methods. In Figure 1 we show two excitation spectra for a Eu(ceDOTA) solution; in (A) the emission was monitored at $589.5 \mathrm{~nm}$ while the dye laser was scanned through the region of the ${ }^{7} \mathrm{~F}_{0} \rightarrow{ }^{5} \mathrm{D}_{0}$ absorption, and in (B) the emission was monitored at $587.0 \mathrm{~nm}$. It is clear that these different emission peaks are associated with excitations associated with different solution species. This can also be seen in the data presented in Figure 2 where the luminescence of $\mathrm{Eu}$ (ceDOTA) is measured following excitation of the two observed ${ }^{7} \mathrm{~F}_{0} \rightarrow{ }^{5} \mathrm{D}_{0}$ absorptions at $579.4 \mathrm{~nm}$ (A) and $579 \mathrm{~nm}$ (B). The two spectral regions shown correspond to the ${ }^{5} \mathrm{D}_{0} \rightarrow$ ${ }^{7} \mathrm{~F}_{1}$ (left) and ${ }^{5} \mathrm{D}_{0} \rightarrow{ }^{7} \mathrm{~F}_{4}$ (right) transitions. Comparison of these results with the luminescence and NMR spectra recorded for related complexes allows one to associate the different peaks in the excitation and luminescence spectrum with the individual isomers. The spectra labeled (A) in Figures 1 and 2 correspond to the twisted square anti-prism structure (TSAP), and (B) denotes the square anti-prism (SAP) isomer. The difference between the two excitation peaks $(0.4 \mathrm{~nm})$ is somewhat larger than that seen for isomers of several Eu(III) complexes with tetraamide-DOTA ligands $(0.12-0.25 \mathrm{~nm}),{ }^{11}$ and only slightly less than has been ascribed to a simple hydration equilibrium $(0.5 \mathrm{~nm}){ }^{12}$

These two isomers both possess approximate $C_{4}$ symmetry. In $C_{4}$ symmetry the $J=1$ state transforms as $\mathrm{A}+\mathrm{E}$; however, allowing for distortions away from $C_{4}$, there should be a maximum of 3 crystal field levels. It is, in fact, possible to fit 

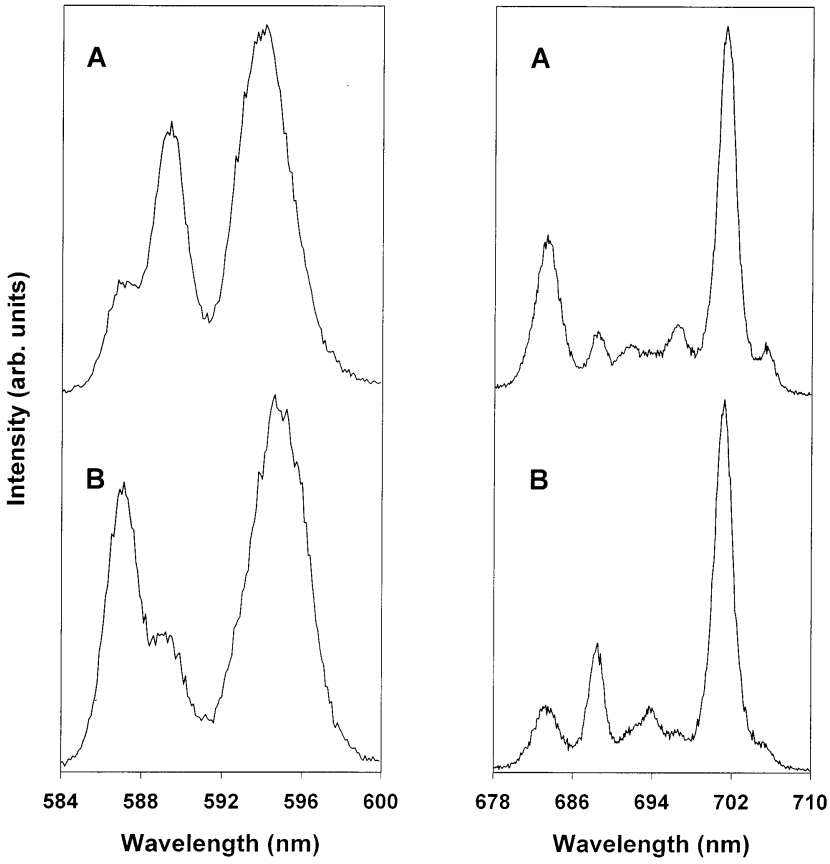

Figure 2. Luminescence spectra for a $0.01 \mathrm{M}$ aqueous solution of Eu(ceDOTA). Excitation was accomplished at $579.4 \mathrm{~nm}$ in (A) and $579 \mathrm{~nm}$ in (B). The spectral regions shown correspond to the ${ }^{7} \mathrm{~F}_{1} \leftarrow$ ${ }^{5} \mathrm{D}_{0}$ (left) and ${ }^{7} \mathrm{~F}_{4} \leftarrow{ }^{5} \mathrm{D}_{0}$ (right) transitions of $\mathrm{Eu}(\mathrm{III})$.

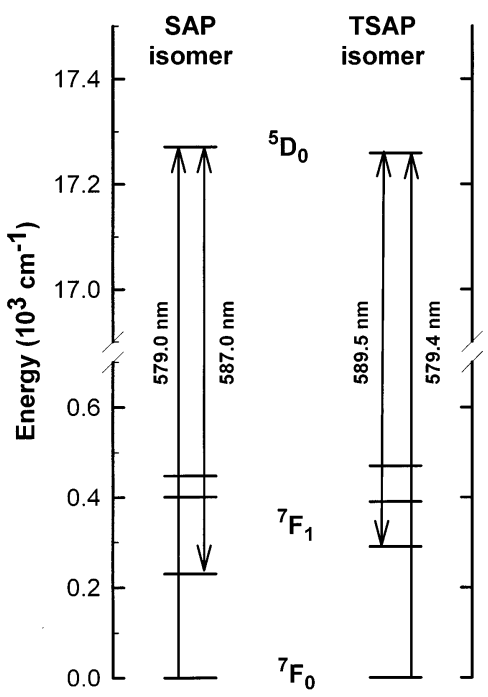

Figure 3. A portions of the approximate Eu(III) energy level diagrams for the SAP and TSAP isomers of Eu(ceDOTA).

the ${ }^{5} \mathrm{D}_{0} \rightarrow{ }^{7} \mathrm{~F}_{1}$ excitation spectra to a sum of three pure Lorentzian line-shapes and to determine the approximate location of the various energy levels. The peak positions derived from the curve-fitting are presented in Figure 3. It should be noted that the lowest ${ }^{7} \mathrm{~F}_{1}$-derived crystal field level (denoted 1a) of the SAP isomer is unusually low in energy $\left(235 \mathrm{~cm}^{-1}\right)$ when compared to other $\mathrm{Eu}(\mathrm{III})$ species. This state is significantly populated (32\%) at room temperature; the lowest crystal field level of the TSAP isomer $\left(290 \mathrm{~cm}^{-1}\right)$ is $25 \%$ populated at room temperature.

There have been several reports in which the temperature dependence of the ${ }^{7} \mathrm{~F}_{0} \rightarrow{ }^{5} \mathrm{D}_{0}$ excitation spectra have been used to probe the solution equilibrium thermodynamics of several $\mathrm{Eu}(\mathrm{III})$ complexes. ${ }^{13-16}$ In these previous studies, it was assumed that the relative populations of the ${ }^{7} \mathrm{~F}_{0}$ states did not change significantly with temperature in the relatively narrow temper- ature range available in aqueous solutions. Due to the low-lying ${ }^{7} \mathrm{~F}_{1}$ crystal field states for the Eu(ceDOTA) isomers, we undertook an analysis in which the explicit temperature dependence of the initial states was taken into account.

We consider the equilibrium depicted in eq 1 between the two structural isomers of Eu(ceDOTA). We rewrite this equilibrium as follows:

$$
\mathrm{SAP}-\mathrm{Eu}(\mathrm{ceDOTA}) \leftrightarrow \mathrm{TSAP}-\mathrm{Eu}(\mathrm{ceDOTA})
$$

For a particular absorption(excitation) transition $\mathrm{n} \rightarrow \mathrm{e}$, we can relate the total concentration, $C$, of each species to the area, $A^{\mathrm{n} \rightarrow \mathrm{e}}$ of the peak associated with the transition as follows:

$$
C_{\mathrm{SAP}} f_{\mathrm{SAP}}^{n}(T)=k_{\mathrm{SAP}}^{\mathrm{n} \rightarrow \mathrm{e}} A_{\mathrm{SAP}}^{\mathrm{n} \rightarrow \mathrm{e}} \quad C_{\mathrm{TSAP}} f_{\mathrm{TSAP}}^{\mathrm{n}}(T)=k_{\mathrm{TSAP}}^{\mathrm{n} \rightarrow \mathrm{e}} A_{\mathrm{TSAP}}^{\mathrm{n} \rightarrow \mathrm{e}}
$$

where $k^{\mathrm{n} \rightarrow \mathrm{e}}$ is a proportionality factor, and $f^{\mathrm{n}}$ denotes the fraction of molecules in the initial state $\mathrm{n}$ at temperature $T$. The equilibrium constant, $K_{\text {eq }}$, may then be expressed as

$$
K_{\mathrm{eq}}=\frac{[\mathrm{TSAP}-\mathrm{Eu}(\mathrm{ceDOTA})]}{[\mathrm{SAP}-\mathrm{Eu}(\operatorname{ceDOTA})]}=\frac{k_{\mathrm{TSAP}}^{\mathrm{n} \rightarrow \mathrm{e}} A_{\mathrm{TSAP}}^{\mathrm{n} \rightarrow \mathrm{e}} f_{\mathrm{SAP}}^{\mathrm{n}}}{k_{\mathrm{SAP}}^{\mathrm{n} \rightarrow \mathrm{e}} A_{\mathrm{SAP}}^{\mathrm{n} \rightarrow \mathrm{e}} f_{\mathrm{TSAP}}^{\mathrm{n}}}
$$

and it follows that

$$
\ln K_{\mathrm{eq}}=\ln \frac{k_{\mathrm{TSAP}}^{\mathrm{n} \rightarrow \mathrm{e}}}{k_{\mathrm{SAP}}^{\mathrm{n} \rightarrow \mathrm{e}}}+\ln \frac{A_{\mathrm{TSAP}}^{\mathrm{n} \rightarrow \mathrm{e}}}{A_{\mathrm{SAP}}^{\mathrm{n} e \mathrm{e}}}-\ln \frac{f_{\mathrm{TSAP}}^{\mathrm{n}}}{f_{\mathrm{SAP}}^{\mathrm{n}}}
$$

We assume here the energy levels given in Figure 3. Assuming a simple Boltzmann distribution, we can write the following expressions for the fractional populations:

$$
\begin{gathered}
{\left[{ }^{7} \mathrm{~F}_{0}\right] \quad f_{\mathrm{SAP}}^{0}=\frac{1}{z_{\mathrm{SAP}}} f_{\beta}^{0}=\frac{1}{z_{\mathrm{TSAP}}}} \\
{\left[{ }^{7} \mathrm{~F}_{1 \mathrm{a}}\right] \quad f_{\mathrm{SAP}}^{1 \mathrm{a}}=\frac{\exp \left(-E_{\mathrm{SAP}}^{1} / k T\right)}{z_{\mathrm{SAP}}} f_{\mathrm{TSAP}}^{1 \mathrm{a}}=\frac{\exp \left(-E_{\mathrm{TSAP}}^{1} / k T\right)}{z_{\mathrm{TSAP}}}}
\end{gathered}
$$

where 1a denotes a particular crystal field state, and the partition functions are given as

$z_{\mathrm{SAP}}=\sum_{i=0}^{3} \exp \left(-E_{\mathrm{SAP}}^{i} / k T\right) \quad z_{\mathrm{TSAP}}=\sum_{i=0}^{3} \exp \left(-E_{\mathrm{TSAP}}^{i} / k T\right)$

The sum runs over all four of the ${ }^{7} \mathrm{~F}_{0}$ and ${ }^{7} \mathrm{~F}_{1}$ states. The enthalpy associated with the equilibrium depicted in eq 1 may be related to the temperature dependence of the equilibrium constant as follows:

$$
\left(\frac{\partial \ln K_{\mathrm{eq}}}{\partial(1 / T)}\right)_{P}=-\frac{\Delta H}{R}
$$

We assume here that the proportionality constants, $k$, are independent of temperature, so that for ${ }^{7} \mathrm{~F}_{0} \rightarrow{ }^{5} \mathrm{D}_{0}$ excitation measurements

$$
\begin{gathered}
\frac{\partial \ln K_{\mathrm{eq}}}{\partial(1 / T)}=\frac{\partial}{\partial(1 / T)}\left[\ln \left(\frac{A_{\mathrm{TSAP}}^{0 \rightarrow 0}}{A_{\mathrm{SAP}}^{0 \rightarrow 0}}\right)+\ln \left(\frac{z_{\mathrm{TSAP}}}{z_{\mathrm{SAP}}}\right)\right] \\
=\frac{\partial R^{0 \rightarrow 0}(T)}{\partial(1 / T)}
\end{gathered}
$$



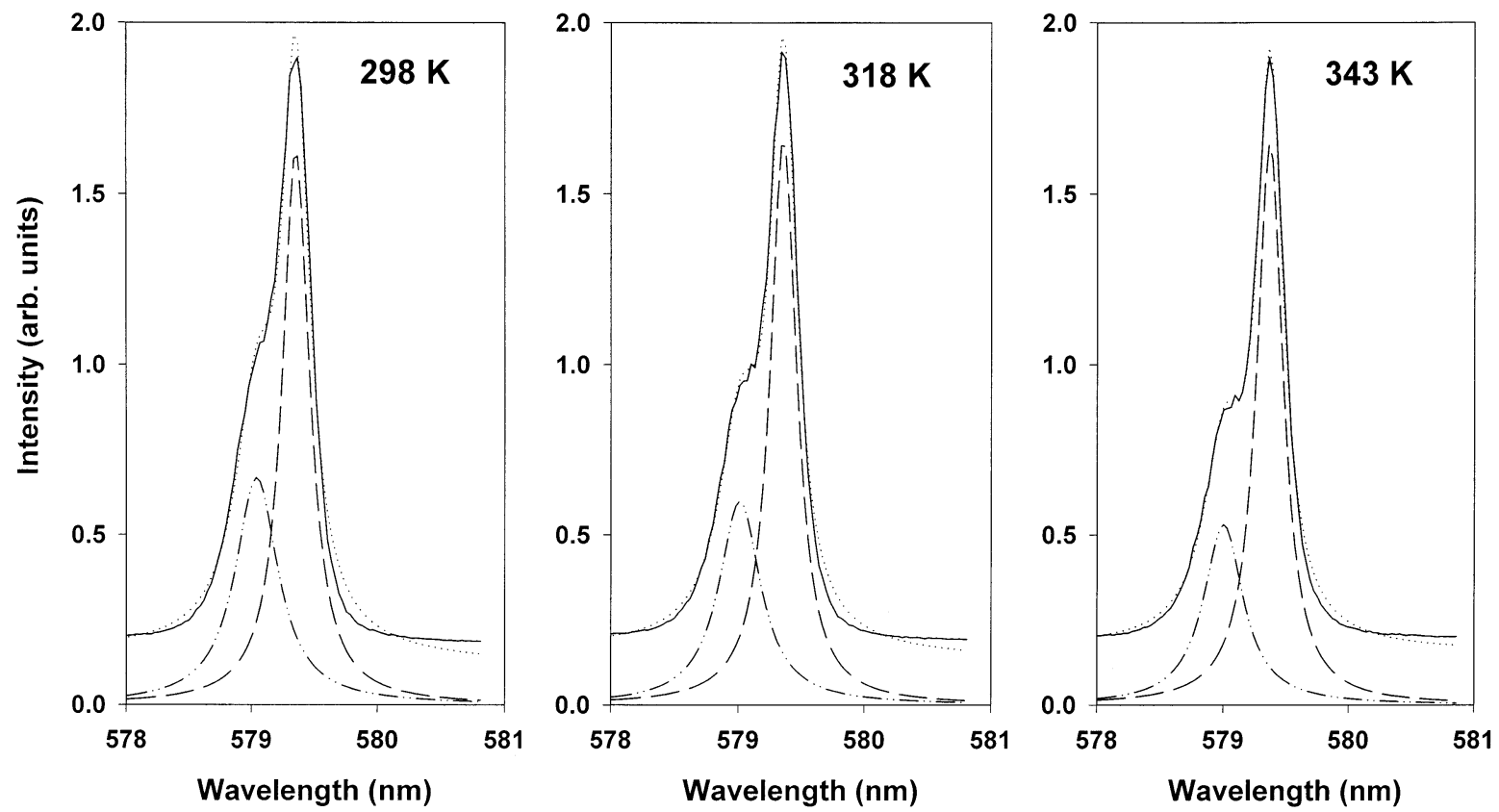

Figure 4. ${ }^{7} \mathrm{~F}_{0} \rightarrow{ }^{5} \mathrm{D}_{0}$ excitation spectra for a $0.01 \mathrm{M}$ aqueous solution of Eu(ceDOTA) at different temperatures. The emission was monitored at $614 \mathrm{~nm}$. The dashed lines are the result of curve fitting the spectra to a sum of two Lorentzian peaks.

and for ${ }^{7} \mathrm{~F}_{1 \mathrm{a}} \rightarrow{ }^{5} \mathrm{D}_{0}$ excitation measurements

$$
\begin{aligned}
\frac{\partial \ln K_{\mathrm{eq}}}{\partial(1 / T)} & =\frac{\partial}{\partial(1 / T)}\left[\ln \left(\frac{A_{\mathrm{TSAP}}^{0 \rightarrow 1 \mathrm{a}}}{A_{\mathrm{SAP}}^{0 \rightarrow 1 \mathrm{a}}}\right)-\ln \left(\frac{\exp \left(-E_{\mathrm{TSAP}}^{1 a} / k T\right) / z_{\mathrm{TSAP}}}{\exp \left(-E_{\mathrm{SAP}}^{1 a} / k T\right) / z_{\mathrm{SAP}}}\right)\right] \\
& =\frac{\partial}{\partial(1 / T)}\left[\ln \left(\frac{A_{\mathrm{TSAP}}^{0 \rightarrow 1 \mathrm{a}}}{A_{\mathrm{SAP}}^{0 \rightarrow 1 \mathrm{a}}}\right)+\ln \left(\frac{z_{\mathrm{TSAP}}}{z_{\mathrm{SAP}}}\right)+\frac{E_{\mathrm{TSAP}}^{1 \mathrm{a}}-E_{\mathrm{SAP}}^{1 \mathrm{a}}}{k T}\right] \\
& =\frac{\partial R^{1 \mathrm{a} \rightarrow 0}(T)}{\partial(1 / T)}
\end{aligned}
$$

Therefore a plot of $R^{0 \rightarrow 0}(T)$ or $R^{1 \mathrm{a} \rightarrow 0}(T)$, as defined in eqs 10 and 11, allows one to estimate the enthalpy from a linear leastsquares determination of the slope.

In Figure 4 we plot several ${ }^{7} \mathrm{~F}_{0} \rightarrow{ }^{5} \mathrm{D}_{0}$ excitation spectra measured at different temperatures. For these measurements, the luminescence was monitored at $605-615 \mathrm{~nm}$, corresponding to the strong emission associated with the ${ }^{5} \mathrm{D}_{0} \rightarrow{ }^{7} \mathrm{~F}_{2}$ transitions for the two isomers. The dashed lines in this figure correspond to curve fits accomplished using Jandel Peak Fit. The peaks were assumed to be pure Lorentzian in shape, so only the peak position and Lorentzian width were varied. As can be seen, although the excitation peaks corresponding to the two isomers are not resolvable, it is clear that the equilibrium shifts toward the TSAP isomer as temperature increases. In Figure 5 we plot the quantity in brackets in eq 10 versus inverse temperature in order to determine $\Delta H$ for this equilibrium. The dashed line in this figure shows the results obtained when the differential temperature-dependent population effects are neglected (i.e., zSAP $=z_{\mathrm{TSAP}}$ ). As can be seen in this plot, inclusion of the variation of initial state thermal population with temperature in this analysis has only a small effect on the slope. Substitution of the slope from this graph into eq 9 yields a value of $+7.5 \pm$ $0.5 \mathrm{~kJ} / \mathrm{mol}$ for $\Delta H$.

Because we are able to identify specific peaks in the ${ }^{7} \mathrm{~F}_{1} \rightarrow$ ${ }^{5} \mathrm{D}_{0}$ excitation spectrum with individual isomers, it is also possible to perform an analysis similar to that described above, using peak areas from curve fits of these peaks. In Figure 6 we

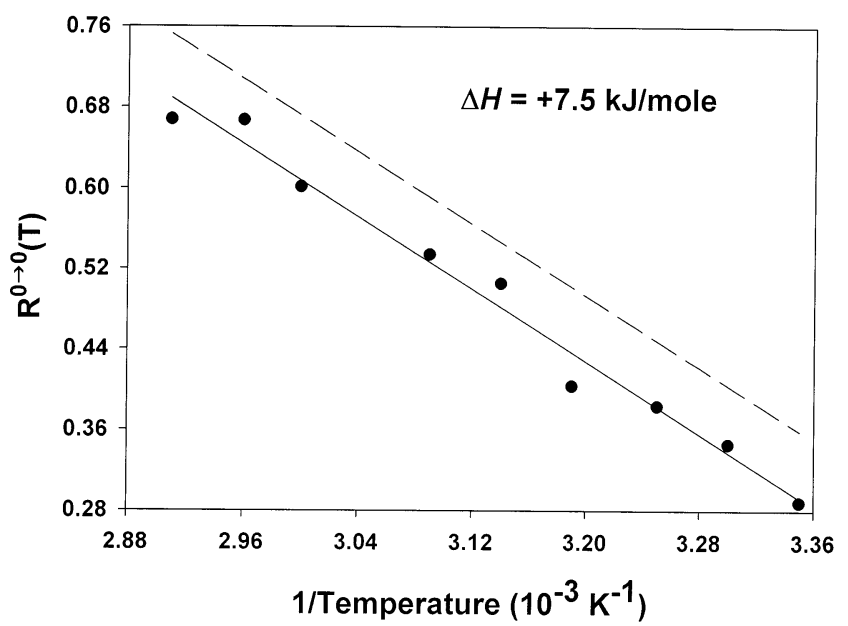

Figure 5. Plot of $R^{0 \rightarrow 0}(T)$ versus inverse temperature. (See eq 10 and text.) The solid line represents the result of a linear least-squares regression, and the dashed line is the result obtained when the differential temperature dependence of the initial state population is neglected.

plot several ${ }^{7} \mathrm{~F}_{1} \rightarrow{ }^{5} \mathrm{D}_{0}$ excitation spectra covering a range of temperatures. The areas of the fairly well-separated Lorentzian peaks at $587.0 \mathrm{~nm}$ (SAP) and $589.5 \mathrm{~nm}$ (TSAP) were determined, and a plot of the quantity in brackets in eq 11 versus inverse temperature is given in Figure 7. Again, the dashed line in this figure corresponds to the neglect of the temperature dependence of the initial state population. From least-squares fitting of these results we obtain a value of $+6.9 \mathrm{~kJ} / \mathrm{mol}$. The effect of the full temperature-dependent analysis is more important for excitations originating from this thermally excited state, but the overall result is within the error associated with the curve fitting procedure $( \pm 0.5 \mathrm{~kJ} / \mathrm{mol})$.

It is interesting to note that the effect of temperaturedependent initial state populations on the analysis presented here is opposite for the two excitation spectra. Since the energy difference between the lowest-lying crystal field state $\left({ }^{7} \mathrm{~F}_{1 \mathrm{a}}\right)$ of the SAP isomer is less than for the TSAP isomer, increasing temperature will decrease the population of the ${ }^{7} \mathrm{~F}_{0}$ state of 

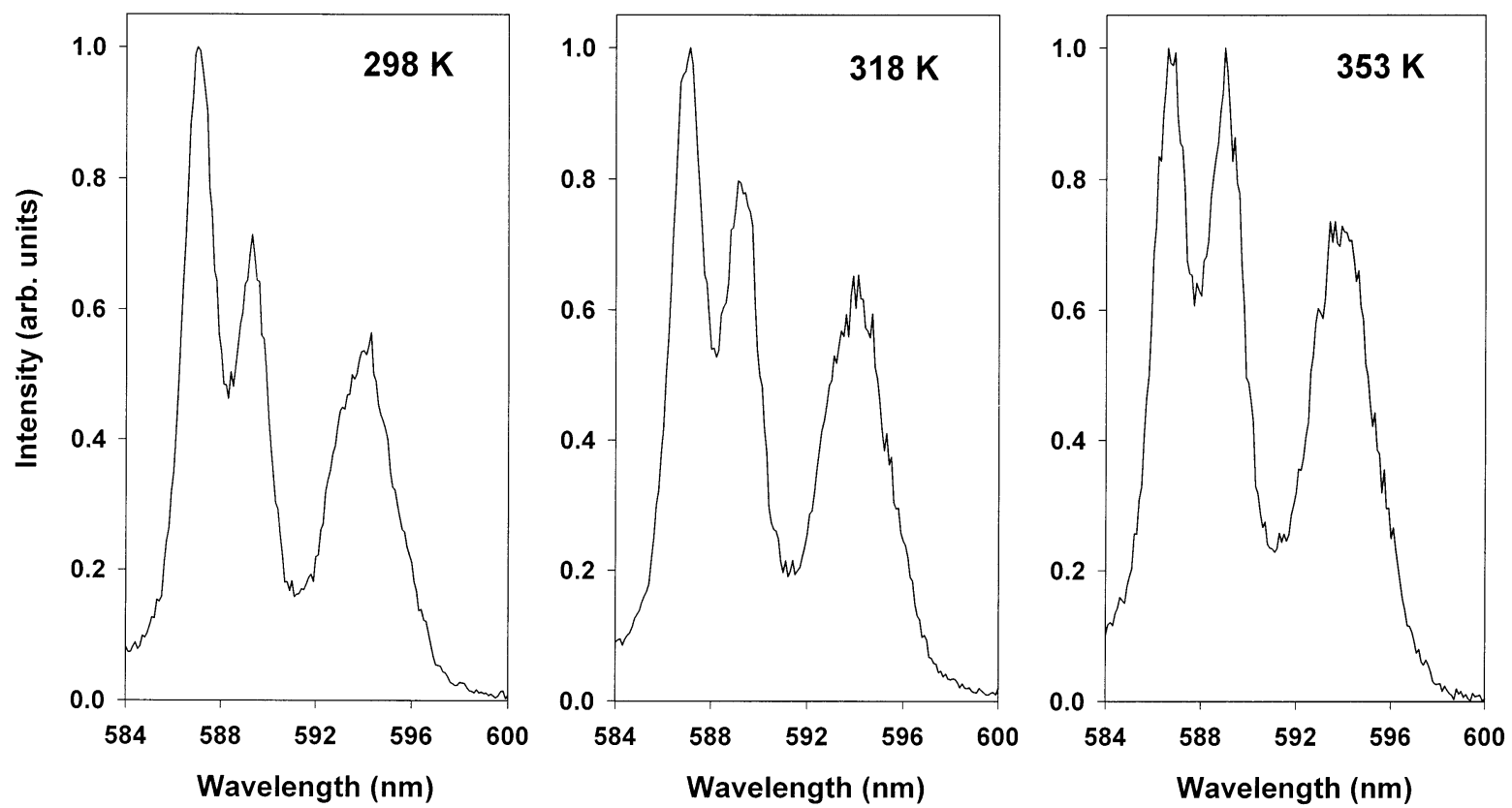

Figure 6. ${ }^{7} \mathrm{~F}_{1} \rightarrow{ }^{5} \mathrm{D}_{0}$ excitation spectra for a $0.01 \mathrm{M}$ aqueous solution of Eu(ceDOTA) at different temperatures. The emission was monitored at $614 \mathrm{~nm}$.

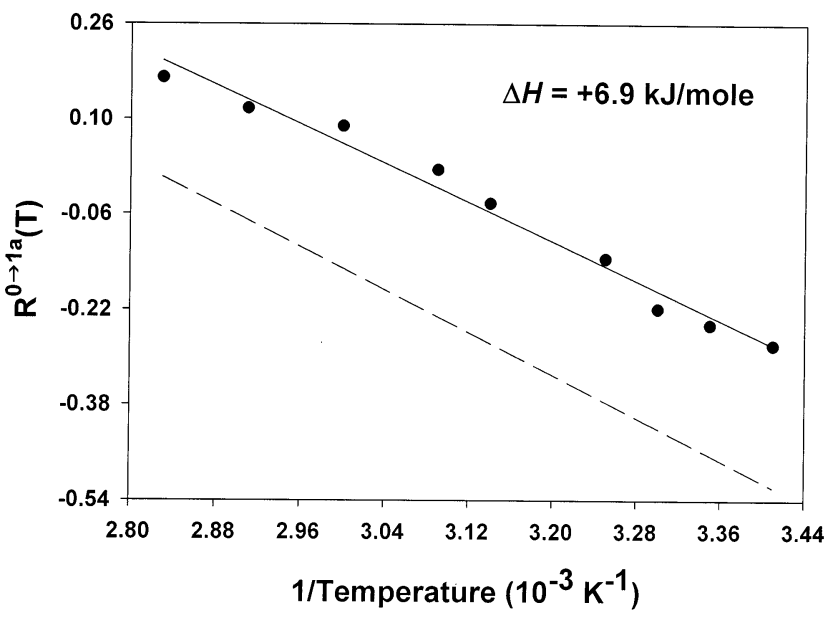

Figure 7. Plot of $R^{1 \mathrm{a} \rightarrow 0}(T)$ versus inverse temperature. (See eq 11 and text.) The solid line represents the result of a linear least-squares regression, and the dashed line is the result obtained when the differential temperature dependence of the initial state population is neglected.

the SAP isomer more than the TSAP isomer, and correspondingly increase the population of the ${ }^{7} \mathrm{~F}_{1 \text { a }}$ state of the SAP isomer more than TSAP isomer. This effect can be seen in the slopes of the least-squares lines in Figures 5 and 7.

Useful information concerning structural differences between the two isomers in equilibrium can also be obtained from examination of the pressure dependence of the excitation spectra. ${ }^{14-17}$ The pressure dependence of the equilibrium constant is related to the difference in molar volume, $\Delta V$, by the following relationship:

$$
\left(\frac{\partial \ln K_{\mathrm{eq}}}{\partial P}\right)_{T}=-\frac{\Delta V}{R T}
$$

As shown previously, ${ }^{15}$ under the assumption that the proportionality constants given in eq 3 are independent of pressure, one can relate the pressure dependence of the equilibrium
TABLE 1: Summary of Thermodynamic Results for SAP $\leftrightarrow$ TSAP Equilibrium

\begin{tabular}{llc}
\hline & \multicolumn{1}{c}{$\mathrm{Eu}(\mathrm{ceDOTA})^{a}$} & $\mathrm{Eu}(\mathrm{DOTMAM})^{b}$ \\
\hline$K_{\mathrm{eq}}$ & $\sim 4$ & $3.3 \pm 0.1$ \\
$\Delta H(\mathrm{~kJ} / \mathrm{mol})$ & $+7.5 \pm 0.5(0 \rightarrow 0)$ & $-3.7 \pm 1$ \\
& $+6.9 \pm 0.5(1 \mathrm{a} \rightarrow 0)$ & \\
$\Delta S(\mathrm{~J} / \mathrm{mol} / \mathrm{K})$ & $+33 \pm 2$ & $-2.6 \pm 3$ \\
$\Delta V\left(\mathrm{~cm}^{3} / \mathrm{mol}\right)$ & $+2.5 \pm 0.1$ & $+1.7 \pm 6$
\end{tabular}

${ }^{a}$ This work (see text). ${ }^{b}$ Ref 6 using NMR techniques.

constant to the peak areas as follows:

$$
\left(\frac{\partial \ln K_{\mathrm{eq}}}{\partial P}\right)_{T}=\left[\frac{\partial}{\partial P}\left(\ln \frac{A_{\mathrm{TSAP}}^{n \rightarrow 0}}{A_{\mathrm{SAP}}^{n \rightarrow 0}}\right)\right]=-\frac{\Delta V}{R T}
$$

so that $\Delta V$ may be determined from a plot of the logarithm of peak area ratios versus pressure. In Figure 8 we plot several ${ }^{7} \mathrm{~F}_{0} \rightarrow{ }^{5} \mathrm{D}_{0}$ excitation spectra at different pressures. The dashed lines in these spectra are the results of curve fits to Lorentzian line-shapes. As seen in these results, increasing pressure results in a small shift of the equilibrium in favor of the presumably more compact SAP isomer. In Figure 9 we plot the results of a series of curve fits in order to determine $\Delta V$ using eq 13. A linear least-squares fit to these data results in a value of +2.5 $\mathrm{cm}^{3} / \mathrm{mol}$. A summary of the numerical results including error estimates are given in Table 1.

\section{Discussion}

The results presented above show that for the Eu(ceDOTA) complex, the SAP isomer is the more compact $\left(\Delta V=V_{\mathrm{TSAP}}-\right.$ $\left.V_{\text {SAP }}>0\right)$, and has the lower enthalpy $(\Delta H>0)$. The equilibrium constant for eq 1 has been estimated from NMR studies to be approximately $4 ;{ }^{7}$ using this value and the enthalpy result, one can calculate a value of $+33 \mathrm{~J} / \mathrm{K} / \mathrm{mol}$ for $\Delta S$. One concludes, therefore, that the SAP isomer is also the more ordered of the two structures. Although appropriate care must be taken in interpreting these equilibrium results in terms of a kinetic process, these data are consistent with the experimental 

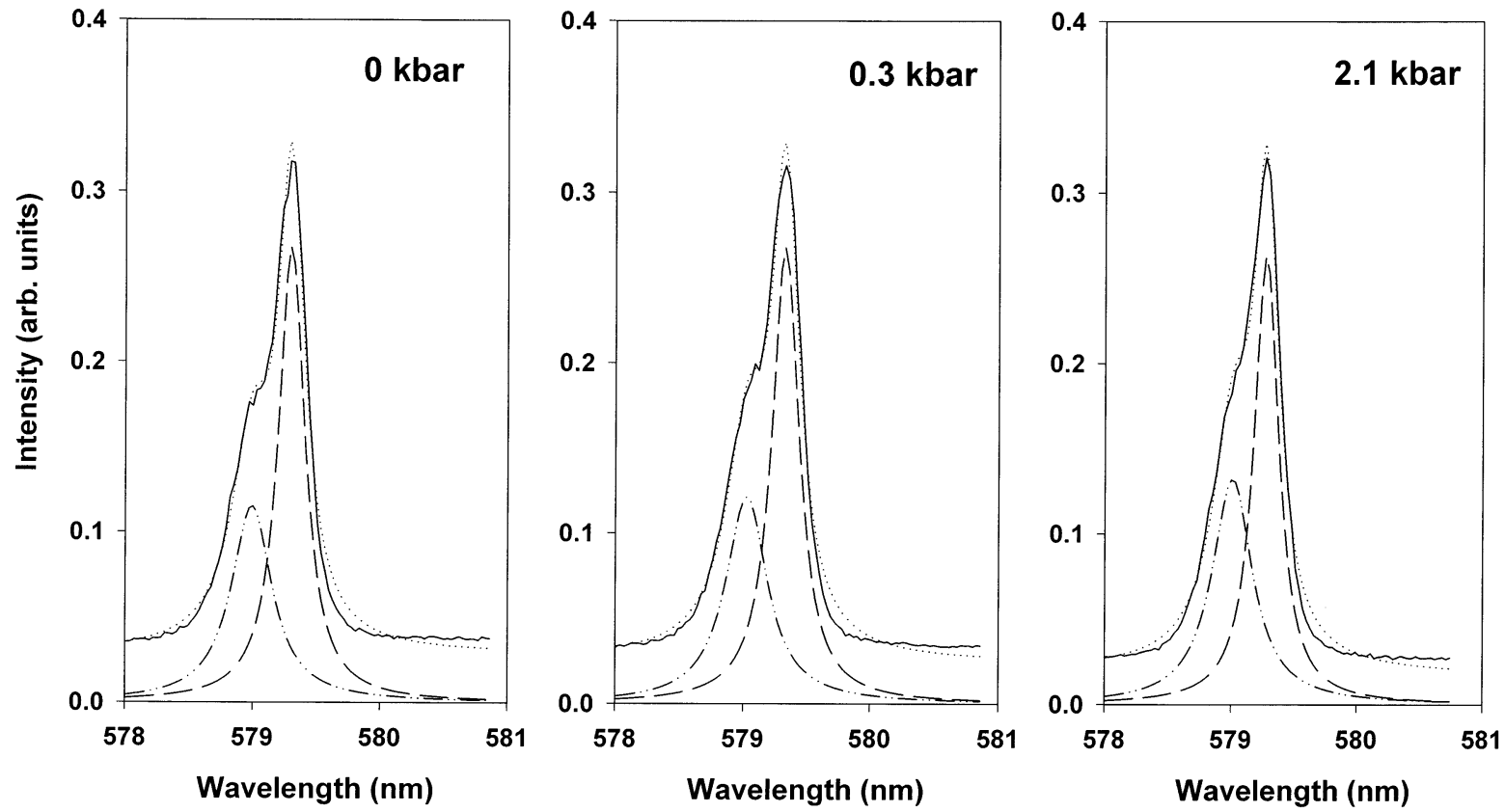

Figure 8. ${ }^{7} \mathrm{~F}_{0} \rightarrow{ }^{5} \mathrm{D}_{0}$ excitation spectra for a $0.01 \mathrm{M}$ aqueous solution of Eu(ceDOTA) under conditions of different solution pressures. The emission was monitored at $614 \mathrm{~nm}$. The dashed lines are the result of curve fitting the spectra to a sum of two Lorentzian peaks.

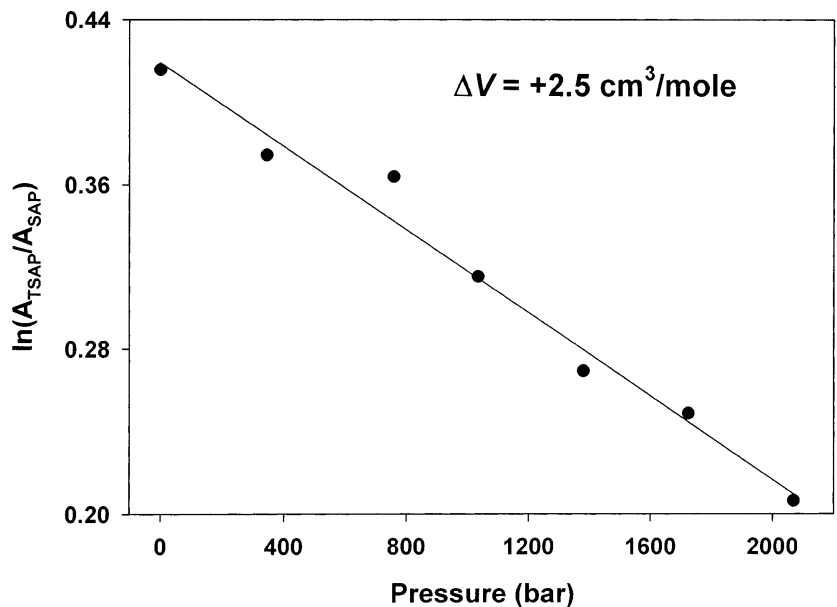

Figure 9. Plot of $\ln \left(A_{\mathrm{TSAP}} / A_{\mathrm{SAP}}\right)$ versus pressure (see eq 13 and text). The dashed line represents a linar least-squares fit to the data.

observation that the TSAP complex of Eu(ceDOTA) exchanges water approximately 50 times more rapidly than the SAP isomer. ${ }^{7}$ It makes sense that the less ordered, less compact, slightly higher energy structure is able to exchange water molecules more easily.

These results should be compared with previous NMR measurements at various temperatures and pressures on a related Eu(III) complex with the $\alpha$-substituted tetraamide dota derivative (DOTMAM) given below. For this species, Dunand et al. ${ }^{6}$ report the values that are also listed in Table 1. Although the equilibrium constants for the two species listed in Table 1 are approximately the same, the equilibrium thermodynamic quantities are quite different in sign and magnitude. Particularly noticeable is the large difference in $\Delta S$ between the two systems. This is not surprising since the pendant arms on the Eu(ceDOTA) complex have a significantly larger number of available conformations in a slightly disordered complex. It is certainly the case that the changes in optical spectra seen for the Eu(ceDOTA) complex, are more evident than the corre- sponding changes seen in NMR experiments for Eu(DOTMAM), leading to values with much less uncertainty.

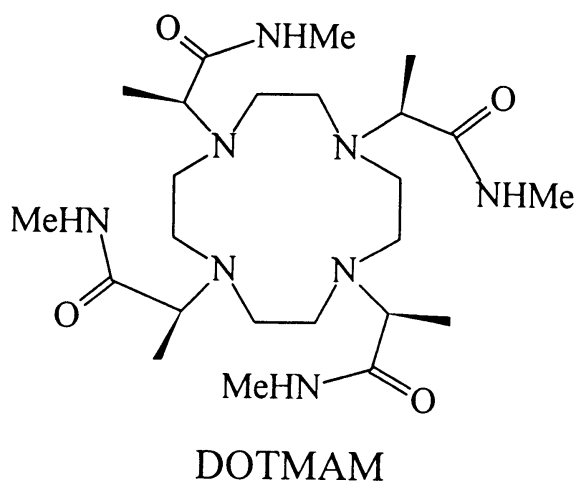

As discussed above, inclusion of the temperature dependence of the initial state populations did not significantly alter the value for enthalpy determined for Eu(ceDOTA), because the major uncertainty in the data and analysis presented here is the error associated with the curve fitting. Depending on the different circumstances, however, this effect can be significant. If one was interested and able to calculate the equilibrium constant from these optical absorption or excitation measurements for $\mathrm{Eu}$ (ceDOTA), for example, the error in ignoring the Boltzmann distribution would be approximately $6 \%$ at room temperature if using the ${ }^{7} \mathrm{~F}_{0} \rightarrow{ }^{5} \mathrm{D}_{0}$ transition, and $20 \%$ if using the ${ }^{7} \mathrm{~F}_{1} \rightarrow$ ${ }^{5} \mathrm{D}_{0}$ spectra. At $80{ }^{\circ} \mathrm{C}$ the errors would be $8 \%$ and $30 \%$, respectively. Obviously, for equilibria involving species in which the crystal field levels are not that different, the effects would be much smaller, and it would also be more difficult to resolve and associate peaks with the different structures.

Acknowledgment is made to EPSRC (D.P.) and the University of Minnesota Duluth for partial support of this work.

\section{References and Notes}

(1) Parker, D. Coord. Chem. Rev. 2000, 205, 109. 
(2) Aime, S.; Botta, M.; Fasano, M.; Terreno, E. Chem. Soc. Rev. 1998, 27,19

(3) Toth, E.; Burai, L.; Merbach, A. E. Coord. Chem. Rev. 2001, 216217,363 .

(4) Caravan, P.; Ellison, J. J.; McMurray, T. J.; Lauffer, R. B. Chem. Rev. 1999, 99, 2293.

(5) Aime, S.; Barge, A.; Batsanov, A. S.; Botta, M.; Castelli, D. D. Fedeli, F.; Mortillaro, A.; Parker, D.; Puschmann, H. Chem. Commun. 2002 1120.

(6) Dunand, F. A.; Dickins, R. S.; Parker, D.; Merbach, A. E. Chem. Eur. J. 2001, 7, 5160

(7) Woods, M.; Aime, S.; Botta, M.; Howard, J. A. K.; Moloney, J. M.; Navet, M.; Parker, D.; Port, M.; Rousseaux, O. J. Am. Chem. Soc 2000, 122, 9781-9792.

(8) Aspinall, H. C. Chem. Rev. 2002, 102, 1807-1850.

(9) Riehl, J. P.; Richardson, F. S. Chem. Rev, 1986, 86, 1-16.
(10) Riehl, J. P.; Richardson, F. S. Methods Enzymol. 1993, 226, 539553

(11) Dunand, F. A.; Merbach, A. E. Inorg. Chem. Commun. 2001, 4, $719-722$.

(12) Graeppi, N.; Powell, D. H.; Laurenczy, G.; Zekany, L.; Merbach, A. E. Inorg. Chim. Acta 1995, 235, 311.

(13) Maupin, C. L.; Smith, K. C.; Riehl, J. P. J. Alloys Compd. 1997, 249, 181-184.

(14) Maupin, C. L.; Logue, M. W.; Leifer, L.; Riehl, J. P. J. Alloys Compd. 2000, 300-301, 101-106.

(15) Maupin, C. L.; Mondry, A.; Leifer, L.; Riehl, J. P. J. Phys. Chem. A 2001, 105, 3071-3076.

(16) Dossing, A.; Sokolnicki, J.; Riehl, J. P.; Legendziewicz, J. J. Alloys Compd. 2002, 341, 150-155.

(17) Gawryszewska, P. P.; Pietraszkiewicz, M.; Riehl, J. P.; Legendziewicz, J. J. Alloys Compd. 2000, 300-301, 283-288. 